\title{
The Fog Computing: Characteristics and Future Directions
}

\author{
Nedhal A. Ben-Eid \\ Computer Department, The Higher Institute for Telecomm. and Navigation, Public Authority for Applied Education \\ and Training (PAAET), Shuwaikh, Kuwait
}

\begin{abstract}
In the last decade, moving computing, control, data storage, and processing to the cloud has been a major trend. But due to the rapidly increasing number of Internet of Things IoT devices/sensors over the world, the cloud was encountered many troubles and limitations, such as high latency, poor quality of service, and location awareness, especially for real-time applications that needs interactivity and fast response. To support this computational demand of real-time latency-sensitive applications of largely geo-distributed IoT devices/sensors, a new computing paradigm named Fog Computing has been introduced [1]. Fog computing is a novel technology that extends and supports cloud computing to reach the optimal performance in the network. Fog computing is acting by pushing the computing and services to the edge of the network. In this paper, we will introduce the Fog Computing term, its architecture is explained, the characteristics are discussed, a comparison with Cloud Computing is examined, and future research directions are mentioned.
\end{abstract}

Keywords: Fog, Cloud, Computing, Internet-of-Things, IoT, Latency, Bandwidth, Real-Time Application

\section{INTRODUCTION}

The Internet of things (IoT) became one of the most important topics in nowadays network technology field. It emerged at homes, streets, offices, organizations, and everywhere in our daily lives. All companies and manufacturers are competing to produce smarter devices that can collect, process, manipulate, and store data with minimal latency, high level of QoS, and minimum operational cost, to help in making the life easier and better.

According to CISCO Annual Internet Report (2018-2023) [5]; there will be 5.3 billion Internet users (66 \% of global population) by 2023, up from 3.9 billion (51\% of global population) in 2018. The number of connected devices will be 29.3 billion (3.6/capita) by 2023, up from 18.4 billion (2.4/capita) in 2018. The connected home applications will be $48 \%$ of M2M share, and car applications will grow the fastest to be 30\% CAGR over the period (2018-2023). Figure 1 shows the growth of connected devices (2018-2023).

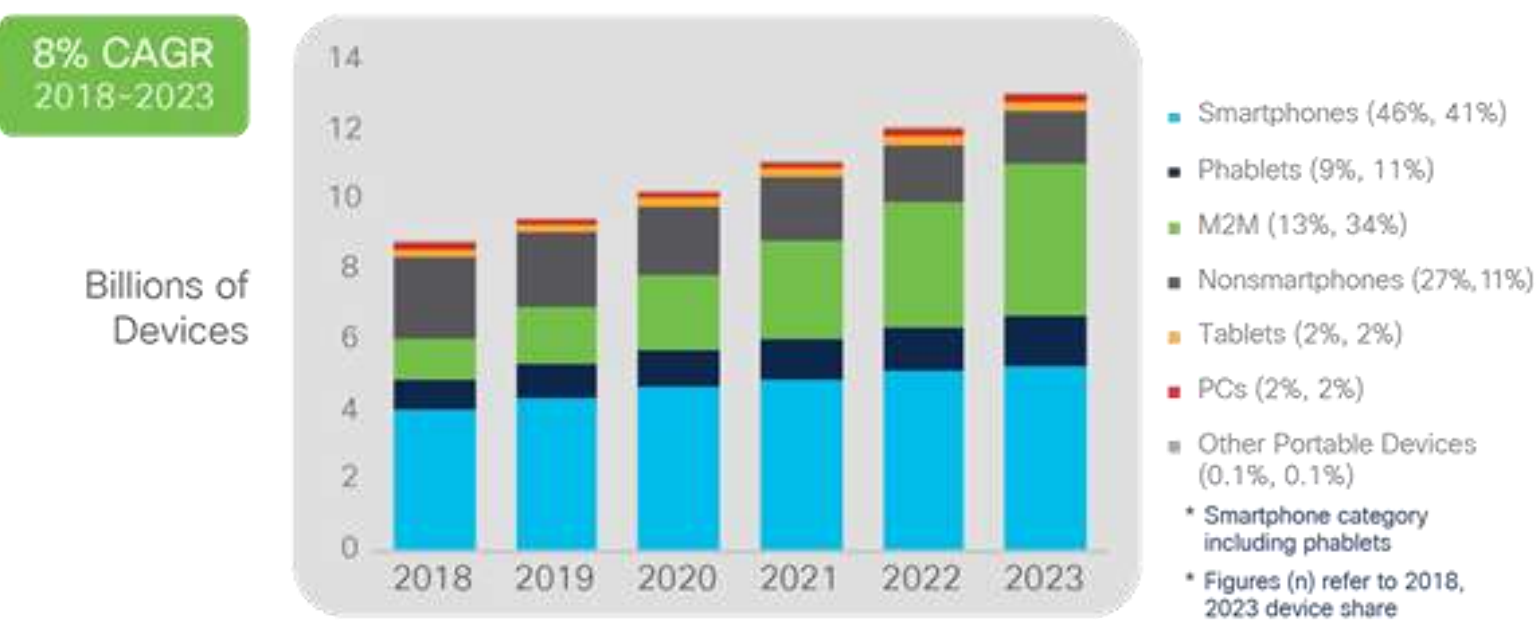

Fig. 1 Global mobile device and connection growth "Cisco Annual Report 2018-2023"

There is an increasingly prominent trend in many organizations to move a substantial portion or even all information technology (IT) operations to an Internet-connected infrastructure known Cloud Computing [14]. It is a model for enabling ubiquitous, convenient, on-demand network access to a shared pool on configurable computing resources that can be rapidly provisioned and released with minimal management effort or service provider interaction [14]. 
The server ends used by IoT are deployed in Cloud for its fair benefits provided to the users [8]. As a whole Cloud computing provides various advantages to its user such as cost efficiency, backup and recovery, easy access to information, quick deployment, automatic software integration, but there are some disadvantages also related to technical issues with internet connection, security issues, limited control and flexibility, dependency and vendor lock-in, and increased vulnerability [6]. Latency is one of the major factors that degrades the performance of cloud since the datacenters are located faraway in the core network, this problem will arise in the real-time applications that needs interactive response.

Due to the rapidly growth of number of IoT devices/sensors technology, like smart phones, smart homes, smart cities, and smart transportation, the smart applications needs resources that can handle and process the collected data effectively and without delay, which cannot be done without the assistance of powerful distributed infrastructure that work with cloud in a coordinative manner.

\section{WHAT IS FOG COMPUTING?}

Fog computing (or Fogging) was introduced by CISCO in 2012 to overcome the limitations and challenges faced by cloud computing. It is a virtualized platform model that process and manipulates data of IoT devices/sensors on the ground (near the edge devices), it extends the cloud computing but not replacing it. Fog computing is a distributed computing paradigm that acts as an intermediate layer in between Cloud data centers and end-user devices [1].

Fog and Cloud Computing are both infrastructure models that provide data processing, computing, storing, and application services to end-users. They are coordinating and managing the processing of data in an orchestration manner to reach the best performance in the network, since all modern applications needs real-time interactions and requires minimum latency and the best quality of service.

To understand how fog computing works, we will explain the architecture of fog computing. In recent years, multiple architecture of fog computing had been suggested, we will explain two of the basic architectures of Fog Computing:

\section{III.Fog COMPUTING ARCHITECTURES}

The fog computing main task is to bring collecting and processing data near the edge, allowing resources to be handled near the devices. To understand the communications and control in the fog network, we will discuss two of the main architectures used in fog computing:

\section{A. Hierarchical Architecture:} It consists of three tiers as illustrated in Figure 1 below:

- $\quad$ Tier 1- IoT/ End devices: This tier includes all sensors and smart devices like smart phones, smart watches, tablets, and all GPS equipped devices. These terminal nodes will send request for services to the fog through fog-terminal interface.

- $\quad$ Tier 2- Fog: This tier includes all network devices like switches, routers, gateways, and access points. These fog nodes provide services and resources to the terminal nodes whenever requested.

- $\quad$ Tier 3-Cloud: This tier is the topmost level in the architecture. It includes servers, routers, and datacenters that have appropriate storage and processing power. These datacenters schedule tasks from fog and process them through fogcloud interface.

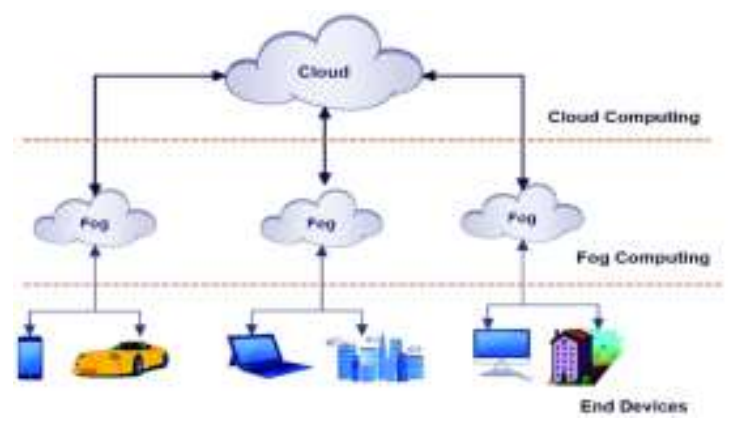

B. The layered Architecture:

Fig. 2 Hierarchical Architecture of Fog Computing

The layered fog architecture consists of six layers:

- $\quad$ Physical and virtualization layer: All IoT devices and sensors collect data and send it for upper layer for processing and filtering. This action is done by physical and virtual nodes.

- $\quad$ Monitoring layer: Node monitoring is performed, to check the working time, battery consumption, temperature, and the application performance.

- $\quad$ Pre-processing layer: The collected data is filtered and cleaned from impurities, then data analysis is applied. 


\section{DOI 10.17148/IJARCCE.2021.10615}

- Temporary storage layer: The analyzed data is stored permanently in this layer, but once it is transferred to the cloud, it will be removed from this layer.

- $\quad$ Security layer: This layer ensures secured and preserved data procedures by applying data privacy, location privacy, usage privacy, data encryption, and data decryption.

- Transport layer: The secured-analysed data is passed through smart network device and then uploaded to the cloud for permanent storage.

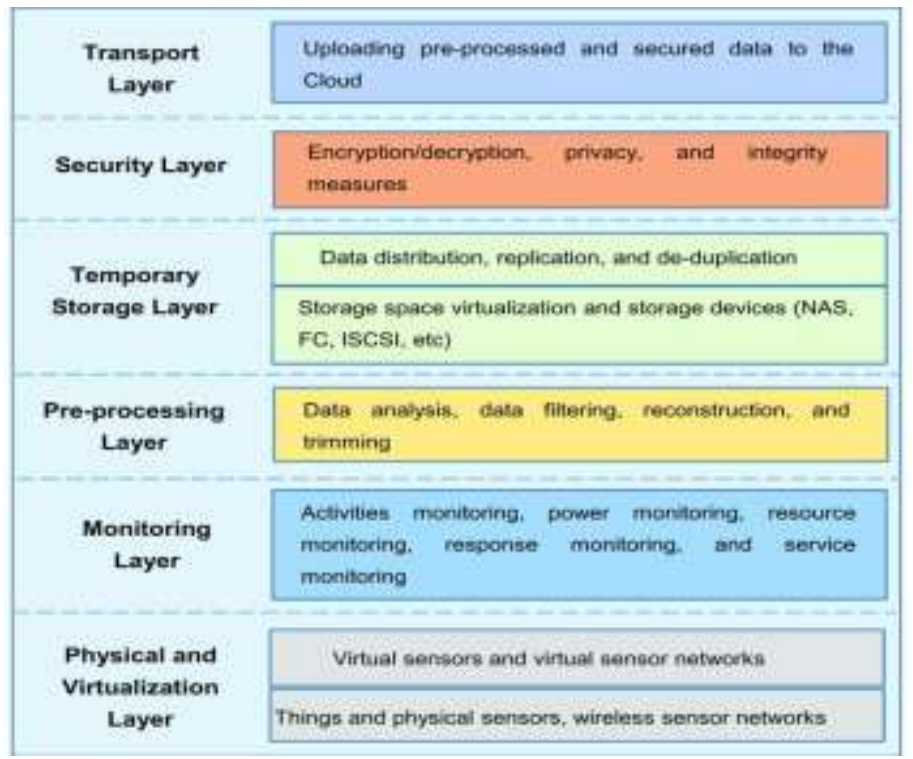

Fig. 3 Layered Architecture of Fog Computing

\section{IV.CHARACTERSTICS OF FOG COMPUTING}

Cloud computing and fog computing share many similar characteristics like adaptable resources (computation, storage, and networking). These resources are building blocks for both cloud and fog computing, signifying that utmost cloud computing technologies can be pragmatic to fog computing. Nevertheless, the new paradigm fog has quite a few exclusive features that makes it diverse from other presence computing architectures [8]. The features of fog computing are discussed and listed below:

- $\quad$ Keeping the Data Close to the User: To avoid data transfer delays, fog permits keeping the data close to the user rather of storing it in distant datacenters.

- $\quad$ Availability and location awareness: The Fog nodes are geographically distributed on large scale distribution to support different types of mobility devices and IoT applications. Fog nodes can be located anywhere like highways and roadways, on a museum floor, on cellular base stations and any other place of interest [8]. All fog nodes can communicate and cooperate even if they are distance apart.

- $\quad$ Scalability: Fog provides the resources anytime needed. As the data gets added, the resource provision is adjusted accordingly. Resources keep on adding, based on the amount of data processing [12].

- $\quad$ Real-Time Interactions: The Fog supports the real-time sensitive application that needs the minimal latency (e.g. online gaming, video streaming, and interactive web browsing).

- Heterogeneity and Compatibility: Devices can interoperate effectively in the fog, even if they come from various providers with different platforms.

- $\quad$ Low latency: The Fog computing is near the edge and IoT devices/sensors, this will shorten the round trip and thus reduce the latency.

- $\quad$ Reduce Operation cost: Fog works as an analyzer, it will filter out the necessary data to be sent to the cloud for processing rather than sending a bulk of data, this process will reduce the consumption of energy and then reducing the operation cost.

- $\quad$ Support for Mobility: This feature enables users to move and change their location freely, with the same quality of service, allowing the preprocessing of data locally before sending it to the datacenters in the cloud.

- $\quad$ Support for IoT: There are billions of IoT devices/sensors over the world that requires the Internet connection and cloud computing, without the Fog assistance, it will be very difficult to handle the huge number of data. Fog Computing will make it easier to manipulate the data in the cloudlets and send only the necessary data to the Cloud. 
Vol. 10, Issue 6, June 2021

DOI 10.17148/IJARCCE.2021.10615

- Greater Business Agility: All customer's needs and requirements for productive organizations work can be done by developing customized applications and tools, which will produce more flexibility and business agility.

- $\quad$ Better Security: Since fog computing processes data near the edge, sensitive data can be manipulated locally instead of sending it to the core network, this will give fog an advantage in the security level. Moreover, we can implement all the security routines and applications that are used in any IT infrastructure in the fog.

\section{COMPARISON OF CLOUD WITH FOG}

Fog computing is quite close to cloud computing in principle. However, the following criteria demonstrate the distinction between these two similar computing technologies. Table 1 outlines the differences between cloud and fog computing. TABLE I COMPARISON BETWEEN CLOUD COMPUTING AND FOG COMPUTING

\begin{tabular}{|l|l|l|}
\hline Criteria & Cloud Computing & Fog Computing \\
\hline Location of Service & at the core network & at the edge \\
\hline Infrastructure & centralized datacenters & distributed cloudlets \\
\hline Number of Server Nodes & few & very large \\
\hline Latency & high & low \\
\hline Response Time & Minutes, days, weeks & milliseconds, seconds, minutes \\
\hline Security & less secure/ undefined & more secure/ definable \\
\hline Location Awareness & not available & available \\
\hline Heterogeneity & low & high \\
\hline Access & through core network/multiple hops & typically through 1-hop wireless \\
\hline Real Time Interaction & not supported/ limited & supported \\
\hline Deployment & fixed, static & dynamic, opportunistic \\
\hline Mobility & not supported/ limited & supported \\
\hline Kind of Last Mile Connectivity & leased line & wireless \\
\hline Virtualization & heavy weight & lightweight \\
\hline Geographic Coverage & global & devices, connected devices, area, cluster \\
\hline
\end{tabular}

\section{CHALLENGES AND FUTURE RESEARCH DIRECTIONS}

We are all witnessing an unstopping evolution toward involving distributed infrastructure located at the edge to execute either innovative services or to enhance the performance of other already existing [15]. This evolution leveraging the highly equipped edge devices that give the final user (not the service provider) the ability to share resources when deploying a new service.

Many experts and companies are doing great efforts to expect what will be the next generation technology in that field. It seams that pushing the computing near the ground (the edge) will be increasing in a harmonic manner with the cloud computing (the core network). Orchestration and interplay between fog and cloud must be available; tasks that needs interactivity and fast response should go to fog, and the tasks that needs huge storage and powerful processing will go to cloud. New research studies may define fog-fog and fog-cloud interfaces to insure smooth transfer of data between the two infrastructures. IoT devices manufacturers should also focus on producing smart devices that can cooperate with each other effectively to have reliable communication using low power consumption.

Many studies and experiments must be directed on measuring the actual bandwidth of fog infrastructure to implement and design ideal system that take into consideration all requirements and features (like latency, bandwidth, operational cost, and location of the fog infrastructure) at the same time. Only few studies have been conducted regarding placing fog nodes in a way considering computing and storage minimization and cost [12].

There is another challenge concerning monitoring fog resources when fog node is accessed by multiple operators, techniques should be devised that take into consideration multi-operator fog monitoring [12].

On the other hand, when talking about the privacy of the collected data in Fog Computing, in some cases (like video camera stream) it attacks the privacy of people by showing their faces and license plate number of the car. Blurring these things before storing and sending the data is very essential to protect people privacy, but this is not present in the Fog Computing Infrastructure model until now.

The security of fog computing in the IoT layer needs more focus and research, current IEE.802.15.4 standard does not completely support keying models and fail to protect acknowledge messages from confidential issues [16]. The security mechanism is not compatible with all the new IoT devices added in the platform, research studies should be done on IoT layer security to be more secured and trustworthy.

Green fog computing is an essential issue that need to be studied and focused on to use minimal energy consumption with the best network performance and quality of service. Renewable energy (like wind, sunlight, etc.) must be used to fuel the fog nodes rather that the regular energy source. 


\section{International Journal of Advanced Research in Computer and Communication Engineering}

Vol. 10, Issue 6, June 2021

DOI 10.17148/IJARCCE.2021.10615

\section{CONCLUSION}

Although cloud computing is popular and used in many IoT applications, it is suffering from many troubles, like high latency, low flexibility, and poor quality of service, especially for real-time applications and big data processing. The rapidly increasing number of IoT applications and devices that needs interactivity nowadays will raise the limitations of cloud computing as well. The adoption of a companion infrastructure that assist the cloud to overcome its troubles is an important issue, and Fog is the most suitable option for that purpose due to its features and characteristics that are explained in this paper.

Fog computing accelerates awareness and response to events by eliminating a round trip to the cloud for analysis. It avoids the need for costly bandwidth additions by offloading gigabytes of network traffic from the core network. It also protects sensitive IoT data by analyzing it inside company walls. Ultimately, organizations that adopt fog computing gain deeper and faster insights, leading to increased business agility, higher service levels, and improved safety [5].

Fog computing was introduced to be the extension of cloud computing, but not a replacement technology, it may push the operation of cloud computing aside near the core network. Nevertheless, a harmony between fog and cloud must be available to have an orchestration that overcomes the limitations of cloud.

Establishing a powerful well-designed paradigm of fog in the intermediate level between the core network and the edge devices is a vital requirement to reach the optimal network performance that satisfies the user needs and visions.

\section{REFERENCES}

[1]. Vighnesh Srinivasa Balaji, Fog Computing and its challenges, K.G Srinivasa, Pankaj Lathar, G. M. Siddesh, The Rise of Fog Computing in the Digital Era, by IGI Global, 2019

[2]. Stojan Kitanov, Toni Janevski, Introduction to Fog Computing, K.G Srinivasa, Pankaj Lathar, G. M. Siddesh, The Rise of Fog Computing in the Digital Era, by IGI Global, 2019

[3]. Gollaprolu Harish, S. Nagaraju, Basavoju Harish, Mazeeda Shaik, "A Review on Fog Computing and its Applications", International Journal of Innovative Technology and Exploring Engineering (IJITEE), ISSN: 2278-3075, vol. 8, Issue: 6C2, April 2019

[4]. C. S. R. Prabhu, "Overview-Fog Computing and Internet-of-Things (IoT)", p. 1, Conference Proceedings: Fog Computing and Internet-of-Things, edited by: C. S. R. Prabhu, 2017

[5]. (2015) The CISCO website. [online]. Available: http://www.cisco.com

[6]. K.P. Saharan, Anuj Kumar, "Fog in Comparison to Cloud: A Survey”, International Journal of Computer Applications (0975 - 8887), vol $122-$ No.3, pp. 10-11, Jul. 2015

[7]. Pooja More1, Juilee Kulkarni, "Fog Computing”, International Research Journal of Engineering and Technology (IRJET), vol 04, Issue 02, pp. 1113-1116, Feb. 2017

[8]. Nida Kauser Khanum, Pankaj Lathar, G.M. Siddesh, Confidentiality and safekeeping problems and Techniques in Fog Computing, The Rise of Fog Computing in the Digital Era, by IGI Global, 2019

[9]. Fog Computing and the Internet of Things: A Review - Scientific Figure on ResearchGate. Available from: https://www.researchgate.net/figure/Fog-computing-is-an-extension-of-the-cloud-but-closer-to-end-devices_fig1_324280213

[10]. Fog Computing: Current Research and Future Challenges - Scientific Figure on ResearchGate. Available from: https://www.researchgate.net/figure/Fog-Computing-Architecture_fig1_324969444

[11]. M. Mukherjee et al., "Security and Privacy in Fog Computing: Challenges," in IEEE Access, vol. 5, pp. 19293-19304, 2017, doi: 10.1109/ACCESS.2017.2749422.

[12]. Umer Iqbal Wani, Ranbir Singh Batth, Mamoon Rashid, "Fog Computing Challenges and Future Directions: A Mirror Review", International Conference on Computational Intelligence and Knowledge Economy (ICCIKE), Dec. 11-12, 2019, Amity University, Dubai, UAE

[13]. Fog Computing and the Internet of Things: A Review - Scientific Figure on ResearchGate. Available from: https://www.researchgate.net/figure/Layered-architecture-of-fog-computing_fig2_324280213

[14]. William Stallings, Foundations of Modern Networking: SDN, NFV, QoE, IoT, and Cloud, Indianapolis, Pearson Education:2016, Indiana, USA

[15]. Yang Yang, Jianwei Huang, Tao Zhang, Joe Weinman, Fog and Fogonomics: Challenges and Practices of Fog Computing, Communication, Networking, Strategy, and Economics, 1 st ed, John Wiley\& Sons, 2020, USA

[16]. https://www.smohanty.org/Publications_Journals/2019/Mohanty_IEEE-CEM_2019-May_Fog-Security.pdf 